\title{
Extracellular vesicles as mediators of neuron-glia communication
}

\section{Carsten Frühbeis*, Dominik Fröhlich, Wen Ping Kuo and Eva-Maria Krämer-Albers}

Department of Molecular Cell Biology, Johannes Gutenberg University Mainz, Mainz, Germany

\author{
Edited by: \\ Martin Stangel, Hannover Medical \\ School, Germany

\section{Reviewed by:} \\ Claudia Verderio, Consiglio Nazionale \\ delle Ricerche, Italy \\ Valentina Bonetto, Istituto di Ricovero \\ e Cura a Carattere Scientifico-Istituto \\ di Ricerche Farmacologiche Mario \\ Negri, Italy

\section{${ }^{*}$ Correspondence:} \\ Carsten Frühbeis, Department of \\ Molecular Cell Biology, Johannes \\ Gutenberg University Mainz, \\ Bentze/weg 3, 55128 Mainz, Germany \\ email: fruehbei@uni-mainz.de
}

In the nervous system, glia cells maintain homeostasis, synthesize myelin, provide metabolic support, and participate in immune defense. The communication between glia and neurons is essential to synchronize these diverse functions with brain activity. Evidence is accumulating that secreted extracellular vesicles (EVs), such as exosomes and shedding microvesicles, are key players in intercellular signaling. The cells of the nervous system secrete EVs, which potentially carry protein and RNA cargo from one cell to another. After delivery, the cargo has the ability to modify the target cell phenotype. Here, we review the recent advances in understanding the role of EV secretion by astrocytes, microglia, and oligodendrocytes in the central nervous system. Current work has demonstrated that oligodendrocytes transfer exosomes to neurons as a result of neurotransmitter signaling suggesting that these vesicles may mediate glial support of neurons.

\section{Keywords: extracellular vesicles, exosomes, neuron-glia communication, oligodendrocytes, microglia, astrocytes, neuroprotection}

\section{INTRODUCTION}

Glia cells are involved in central nervous system (CNS) function, development, and maintenance, which all require intense cell-cell communication between glia and neurons. Intercellular communication can be mediated through direct cell-cell contact or paracrine action of secreted molecules. In the past few years, a novel mode of interaction relying on the exchange of extracellular vesicles (EVs) between cells has become evident. Various cell types release EVs of different origin into their environment, which have the potential to transfer a collection of biomolecules between cells locally or over longer distances (Thery, 2011; Raposo and Stoorvogel, 2013). Glia and neurons secrete EVs and the recent literature implicates that intercellular communication by EVs has versatile functional impact in the CNS (Chivet et al., 2012; Frühbeis et al., 2012; Prada et al., 2013; Sharma et al., 2013).

Extracellular vesicles comprise shedding microvesicles (MVs), exosomes, and apoptotic bodies, which differ in size, cargo, membrane composition, and origin. Apoptotic bodies are released during apoptosis, whereas the other types of vesicles are derived from healthy cells (Cocucci et al., 2009; Thery et al., 2009). A mixture of EVs is detectable in virtually all body fluids and to date it is challenging to clearly discriminate the different types, as some classifying criteria are overlapping (Gould and Raposo, 2013). While MVs directly pinch off from the plasma membrane and are heterogeneous in size (up to $1000 \mathrm{~nm}$ in diameter), exosomes originate from the endosomal system and exhibit a regular shape (50$100 \mathrm{~nm}$ in diameter). Exosomes correspond to the intraluminal vesicles of multivesicular bodies (MVBs), hence their generation involves sorting at the level of the endosomal limiting membrane mediated by the ESCRT (endosomal sorting complex required for transport) machinery (Simons and Raposo, 2009; Baietti et al., 2012) or is assisted by the sphingolipid ceramide and tetraspanins (Trajkovic et al., 2008; Buschow et al., 2009). Fusion of MVBs with the plasma membrane releases exosomes and is controlled by Rab GTPases such as Rab27 in epithelial cells and Rab35 in oligodendrocytes (Hsu et al., 2010; Ostrowski et al., 2010). Exosomes carry characteristic lipids, RNA species, biogenesis-related proteins ( Tsg 101 and Alix are classic markers), tetraspanins, integrins, heat shock proteins, and cell type specific components. On the other hand, they exclude proteins of other intracellular compartments such as the endoplasmic reticulum and mitochondria (Kalra et al., 2012; Raposo and Stoorvogel, 2013). Less is known about the specific composition and biogenesis of MVs. Interestingly, the molecular machinery for MV generation may necessitate factors also involved in exosome generation (Nabhan et al., 2012).

This review summarizes characteristic properties and functions of EVs emphasizing glial EVs in the CNS and in particular the role of oligodendroglial exosomes in neuron-glia communication.

\section{BIOLOGICAL FUNCTIONS OF EVs}

Since their discovery, several physiological and pathological functions have been ascribed to EVs. Reticulocytes utilize exosome release to eliminate obsolete internal membranes during cell maturation (Harding et al., 2013). Furthermore, RNAs are transported by EVs from cell to cell and can modulate gene expression in the recipient cell. After transfer, mRNAs are translated leading to a new set of proteins in the target cell and miRNAs inhibit the expression of resident proteins (Valadi etal., 2007; Skog et al., 2008; Zhang etal., 2010). In the immune system, antigen presenting cells (APCs) secrete exosomes bearing MHC-peptide complexes, which can activate T-cells, suggesting a role of exosomes in the adaptive immune response. On the other hand, tumor exosomes can induce anti-tumor responses but are also able to facilitate tumor development by suppressing immune responses, stimulating tumor growth, invasion, angiogenesis, and metastasis (Bobrie et al., 2011; Luga et al., 2012; Peinado et al., 2012). 
Exosomes also have been implicated in morphogen secretion and thus may mediate evolutionary conserved developmental processes. Exosomes derived from human and Drosophila cells carry Wnt in association with its cargo receptor Evi/Wls (Evenness Interrupted/Wntless) on the surface and induce Wnt signaling in target cells (Gross et al., 2012; Gross and Boutros, 2013). At the Drosophila larval neuromuscular junction pre-synaptic release of exosomes containing Evi/Wls is required for Wnt transmission to the post-synapse (Korkut et al., 2009; Koles et al., 2012). Moreover, synaptotagmin 4 (Syt4) is transferred via exosomes from pre-synaptic terminals to post-synaptic muscles in turn enabling retrograde Syt4 signaling and synaptic growth (Korkut et al., 2013).

In the mammalian nervous system, cortical neurons release exosomes from somatodendritic compartments. Synaptic glutamatergic activity mediates the rise in post-synaptic calcium levels triggering exosome secretion. As neuronal exosomes carry AMPA receptor subunits, they might play a role in synaptic plasticity by regulating the number of AMPA receptors in the post-synaptic membrane (Lachenal etal., 2011; Chivet et al., 2013). Exosomes thus may be implicated in transsynaptic communication in vertebrates and invertebrates. Intercellular transfer of exosomes may be relevant for pathology in several neurodegenerative diseases, since pathogenic proteins such as prions, $\beta$-amyloid peptide, superoxide dismutase, $\alpha$ synuclein, and tau are released from cells in association with EVs (Bellingham et al., 2012; Schneider and Simons, 2012). These vesicles are assumed to spread the pathogenic proteins throughout the tissue. Moreover, EVs derived from glioma cells carry oncogenic EGFRvIII, RNA, and angiogenic factors. They promote cell transformation and modulate the tumor environment to improve tumor growth (Al-Nedawi et al., 2008; Skog et al., 2008).

\section{MICROGLIA-DERIVED EVs}

Microglia, the resident macrophages of the brain, maintain tissue homeostasis, provide the first line of defense during infection and brain injury, and promote tissue repair. In pathological conditions resting microglia polarize toward a M1 (pro-inflammatory) or M2 (pro-regenerative) phenotype largely defined by the profile of secreted cytokines (Hanisch and Kettenmann, 2007; Saijo and Glass, 2011). Microglia bud MVs of irregular shape and size $(0.1-1 \mu \mathrm{m})$ from their plasma membrane characterized by high levels of externalized phosphatidylserine. Upon ATP stimulation of $\mathrm{P}_{2} \mathrm{X}_{7}$ receptors, reactive microglia release MVs carrying the pro-inflammatory cytokine interleukin- $1 \beta$ (IL-1 $\beta$ ), the IL- $1 \beta$ processing enzyme caspase- 1 , and the $\mathrm{P} 2 \mathrm{X}_{7}$ receptor (Bianco et al., 2005). The budding of MVs is facilitated by externalization of acid sphingomyelinase, which induces membrane curvature by locally increasing ceramide levels in the outer leaflet of the plasma membrane (Bianco et al., 2009). The authors suggest that when IL-1 $\beta$ and $\mathrm{P}_{2} \mathrm{X}_{7}$ containing MVs approach tissue areas with high external ATP levels, $\mathrm{MV}$-associated $\mathrm{P}_{2} \mathrm{X}_{7}$ receptors become activated, followed by IL- $1 \beta$ processing and release from MVs. This pathway may induce and propagate inflammatory reactions throughout the brain (Prada et al., 2013).

Microglia-derived MVs can transmit inflammatory signals to recipient microglia, which then upregulate the co-stimulatory molecule CD86 and express pro-inflammatory genes like IL$1 \beta$, IL-6, inducible nitric oxide synthase, and cyclooxygenase-2 (Verderio et al., 2012). MVs derived from all major types of neural cells and in particular MVs carrying myeloid markers are detectable in the rodent and human CSF under normal conditions. In the inflamed brain, in cases of multiple sclerosis in humans and experimental autoimmune encephalomyelitis (EAE) in mice, the amount of MVs increases dramatically depending on disease severity and the extent of microglia activation. Injection of MVs into the brain of mice with subclinical EAE recruits inflammatory cells to the injection site. However, acid sphingomyelinase deficient mice, which are impaired in MV production, are largely protected from EAE. Intriguingly, FTY720, an oral drug for the treatment of multiple sclerosis, reduces the amount of microglial MVs in the CSF of EAE mice. Microglial MVs thus seem to enforce inflammation in neuroinflammatory diseases such as multiple sclerosis. They may represent promising diagnostic markers or even therapeutic targets of brain inflammation (Colombo et al., 2012).

Intriguingly, microglia-derived MVs can interact with neurons and stimulate spontaneous and evoked excitatory transmission in vitro and after injection in vivo. Hippocampal neurons exposed to MVs show an increase in miniature excitatory postsynaptic current (mEPSC) frequency without changes in mEPSC amplitude. MVs affect the pre-synaptic site of the excitatory synapse by increasing the release probability of synaptic vesicles through induction of ceramide and sphingosine synthesis. Thus, microglial MVs appear to modulate synaptic activity and enhance neurotransmission (Antonucci et al., 2012; Turola et al., 2012).

Besides MVs, microglia release exosomes with a protein content analogous to $\mathrm{B}$ cell- and dendritic cell-derived exosomes (Potolicchio et al., 2005). For example, MHC class II is packed into exosomes and its amount is increasing upon stimulation with interferon- $\gamma$. However, whether this is instrumental in antigen presentation and brain immunity is open. In addition, microglial exosomes comprise aminopeptidase N (CD13), which cleaves opioid receptor-binding enkephalins. They also carry enzymes for anaerobic glycolysis and the monocarboxylate transporter 1 (MCT1), potentially delivering energy substrates to target cells, and the insulin degrading enzyme (IDE), which can also degrade the $A \beta$ peptide (Tamboli et al., 2010). It has been suggested that microglial exosome release does not occur constitutively and is induced by Wnt3a, which in turn becomes included in these vesicles (Hooper et al., 2012).

\section{ASTROCYTE-DERIVED EVs}

Astrocytes are multifunctional interactive cells. They are part of the blood brain barrier (BBB), control the extracellular ion balance, provide trophic support, and participate in repair and scarring processes after CNS injury. Similar to microglia, MV shedding from astrocytes is evoked by the ATP-triggered activation of $\mathrm{P}_{2} \mathrm{X}_{7}$ receptors and subsequent action of acid sphingomyelinase (Bianco et al., 2009). Moreover, astrocytes release vesicles from the cell surface that can be up to $8 \mu \mathrm{m}$ in size and carry intact mitochondria, and lipid droplets (Falchi et al., 2013). Astrocytederived EVs are heterogeneous in their composition and have been ascribed beneficial and pathological functions. MVs and exosomes with proposed physiological functions carry $\mathrm{Hsp} / \mathrm{Hsc70}$ 
and synapsin I implicated in neuroprotection (Taylor et al., 2007; Wang et al., 2011), factors modulating angiogenesis such as FGF-2, VEGF, endostatin, and PEDF (Proia et al., 2008; Hajrasouliha et al., 2013), matrix metallo-proteinases mediating extracellular matrix proteolysis (Sbai et al., 2010), and nucleoside triphosphate diphosphohydrolases (NTDPases; Ceruti et al., 2011). The precise action of these EVs on the level of the target cells, however, remains to be determined.

Astrocyte-derived EVs have been implicated in the propagation of pathogenic proteins in neurodegenerative disorders. Astrocytes expressing mutant SOD1 (copper-zinc superoxide dismutase) secrete increased amounts of exosomes, which carry mutant SOD1. These vesicles can transfer mutant SOD1 to cultured neurons and induce motor neuron death suggesting a role of EVs in the pathogenesis of amyotrophic lateral sclerosis (ALS; Basso etal., 2013). Furthermore, exposure of amyloid peptide to astrocytes triggers the release of pro-apoptotic exosomes that include ceramide and PAR4 (prostate apoptosis response 4). These exosomes are taken up by astrocytes and promote their apoptosis suggesting that exosome-mediated astrocyte death may contribute to neurodegeneration in Alzheimer's disease (Wang et al., 2012). Exosome-mediated miRNA transfer from astrocytes to neurons has been suggested to participate in neurodegeneration in HIV-associated neurological disorders ( $\mathrm{Hu}$ et al., 2012). Treatment of cultured astrocytes with pathogenic HIV Tat (trans-activator of transcription) protein and morphine triggers shuttling of miR-29b via exosomes to neuronal cells which results in decreased PDGF-B expression and neuronal viability.

\section{OLIGODENDROCYTE-DERIVED EXOSOMES AND THEIR ROLE IN AXON-GLIA COMMUNICATION}

Oligodendrocytes produce the myelin sheath facilitating impulse conduction. Myelinating oligodendrocytes and axons constitute a sophisticated functional unit based on continuous mutual interaction (Nave, 2010). Hence, maintenance of axonal integrity depends on support from oligodendrocytes. Recent work suggests that this trophic function of oligodendrocytes may relate to the transfer of exosomes from oligodendrocytes to neurons (Frühbeis et al., 2013; Lewis, 2013), in addition to the supply of glycolytic substrates such as lactate (Fünfschilling et al., 2012; Lee et al., 2012). Oligodendrocytes release exosomes that include genuine myelin proteins such as PLP, CNP, MAG, and MOG as well as the NAD-dependent deacetylase sirtuin-2, glycolytic enzymes, and typical exosome-associated proteins such as tetraspanins and heat-shock proteins (Krämer-Albers et al., 2007).

Oligodendrocyte-derived exosomes are internalized by a subset of MHC class II negative microglia via macropinocytosis and are subsequently degraded without provoking any response (Fitzner et al., 2011). In addition, they have been suggested to negatively regulate myelin synthesis in an autocrine fashion (Bakhti et al., 2010). However, in myelinated fibers in situ, PLP-positive MVBs and their fusion profiles were observed in the innermost non-compacted wrapping of the myelin sheath (Figure 1A) implying that exosomes are released into the periaxonal space and involved in axon-glia interaction (Frühbeis et al., 2013). Indeed, the secretion of exosomes from oligodendrocytes is regulated by neurotransmitter signaling. Active neurons release glutamate that provokes $\mathrm{Ca}^{2+}$ entry through oligodendroglial ionotropic glutamate receptors, mostly of the NMDA subtype. This results in elevation of cytosolic $\mathrm{Ca}^{2+}$ levels and triggers exosome secretion. In turn, neurons internalize oligodendrocyte-derived exosomes by clathrin and dynamin-dependent endocytosis at both somatodendritic and axonal compartments (Figure 1B). The uptake appears selective since astrocytes and oligodendrocytes internalize these exosomes to a minor extent. After internalization, the cargo of oligodendroglial exosomes can be functional in the target neuron. The ectopically expressed enzyme Cre-recombinase is packed into exosomes and activates a reporter in the recipient neuron in vitro and, importantly, also after injection of Cre-bearing
A

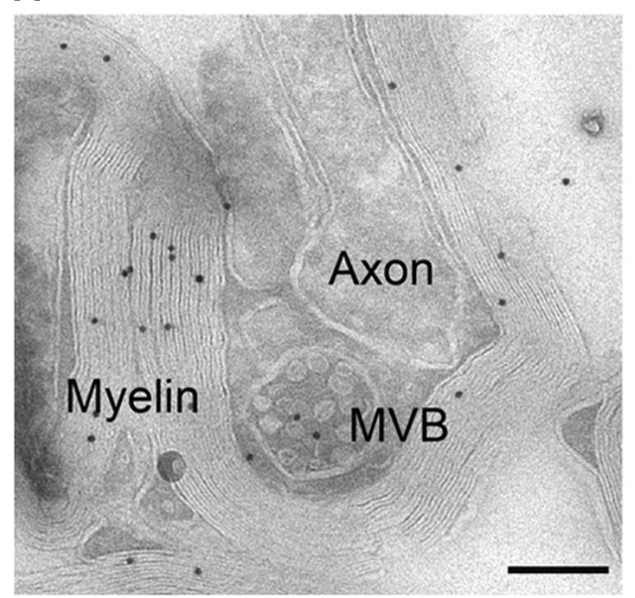

FIGURE 1 | Adaxonal localization of MVBs and uptake of oligodendroglial exosomes by neurons. (A) Electron micrograph of myelinated axons in the optic nerve labeled with antibodies against PLP
B

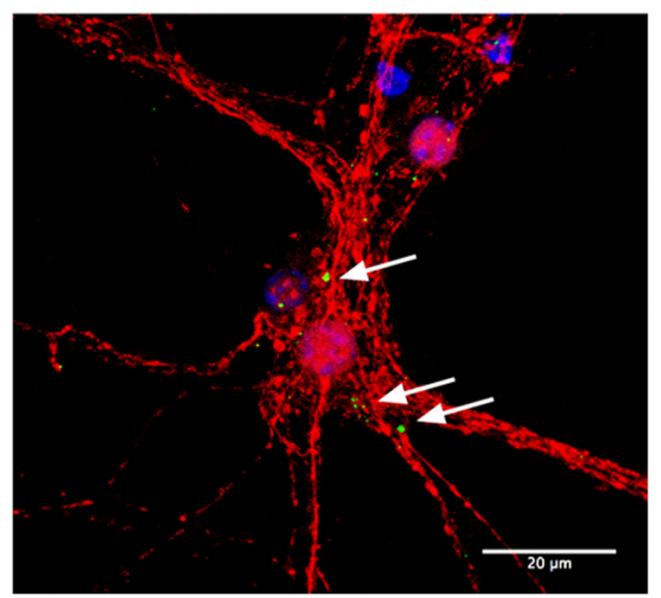

(scale bar $200 \mathrm{~nm}$, courtesy of Wiebke Möbius). (B) Confocal stack of primary cortical neurons (red) that internalized exosomes, labeled with PLP-EGFP and SIRT2-EYFP (green). Nuclei are blue, scale bar $20 \mu \mathrm{m}$. 


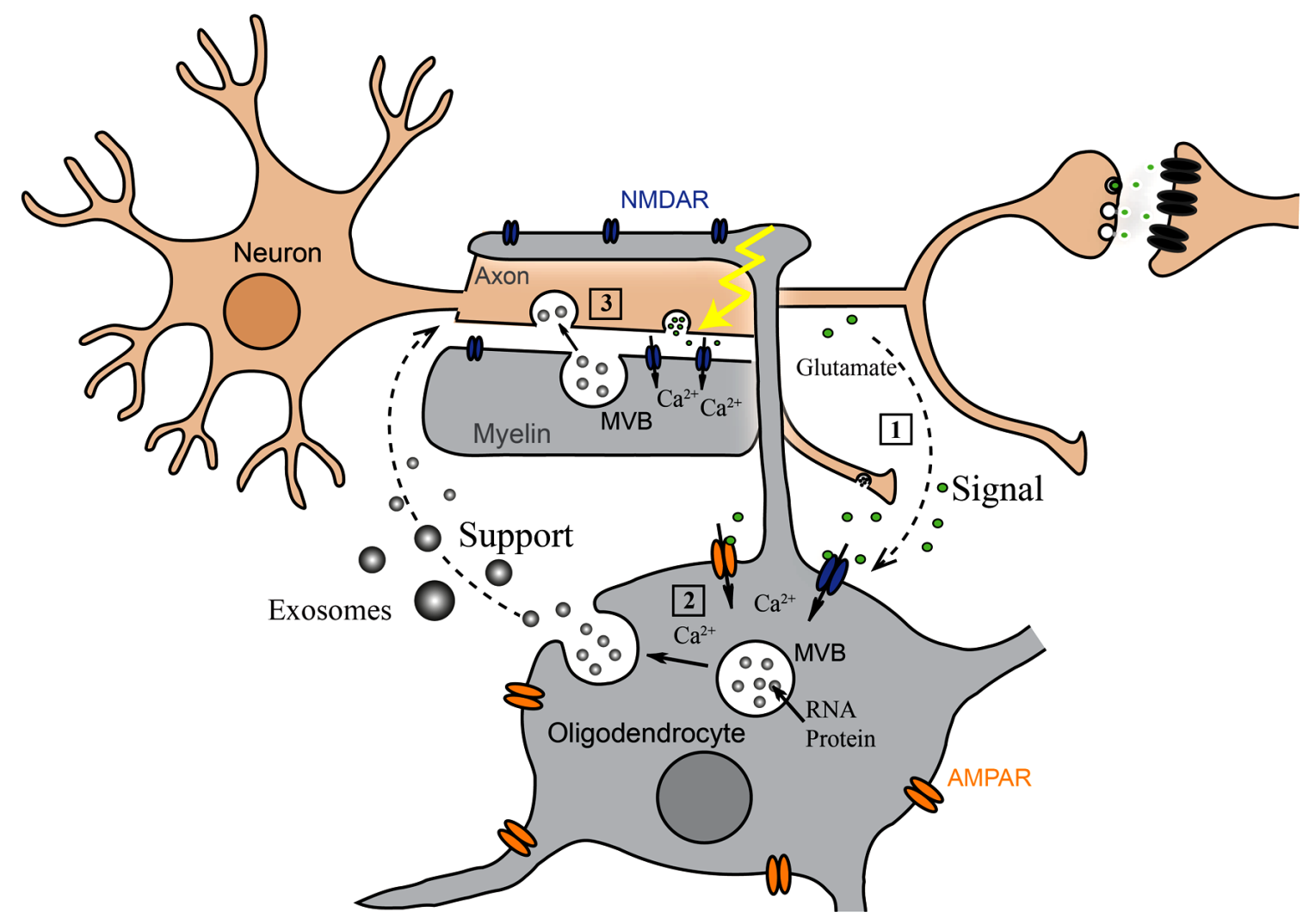

FIGURE 2 | Oligodendroglial exosomes in neuron-glia communication. (1) Electrically active axons release glutamate that provokes Ca $2+$ entry through oligodendroglial glutamate receptors. (2) Elevation of intracellular $\mathrm{Ca}^{2+}$ levels triggers exosome release from oligodendrocytes. (3) Neurons internalize exosomes and use their cargo.

exosomes into the mouse brain. Moreover, oligodendroglial exosomes improve the metabolic activity of cultured neurons under cell stress. In brief, this suggests a model where active neurons signal to oligodendrocytes and demand the delivery of supportive biomolecules via exosomes (Figure 2). Oligodendrocytes can then utilize these vesicles to locally transfer metabolites, protective proteins, glycolytic enzymes, mRNA, and miRNA to axons, which may maintain axonal integrity (Frühbeis et al., 2013). In the peripheral nervous system, evidence suggests that myelinating Schwann cells shuttle supportive cargo to axons by vesicular means facilitating regeneration of axons after injury (Lopez-Verrilli and Court, 2012).

Oligodendroglial trophic support is impaired in CNP and PLP null mice resulting in progressive axonal degeneration (Nave and Trapp, 2008). Since both proteins are components of oligodendroglial exosomes, glial support of axons may be linked to the transfer of substances by exosomes. Lack of these proteins could negatively affect exosome secretion influencing the supply of axons with trophic factors. Indeed, exosome secretion from oligodendrocytes deficient in CNP and PLP is impaired (Frühbeis, Krämer-Albers, unpublished data).

Future work needs to address the identification of surface molecules and target cell receptors mediating vesicle internalization and, moreover, the analysis of functional components which convey support to neurons. Oligodendroglial exosomes include several substances with potentially beneficial activities including stress alleviating proteins and, importantly, mRNA and miRNA. It will be interesting to investigate if RNA transfer via exosomes results in local translation at axonal sites.

\section{CONCLUSION}

Several studies attribute pathological and physiological functions to glial EVs. These vesicles may spread pathogenic factors, promote inflammation, influence neurotransmission, and support neurons. Exosomes secreted by oligodendrocytes transport cargo to neurons and may contribute to axonal integrity. To date, most concepts rely on in vitro data and future work will necessitate genetic models to underscore their importance. Nevertheless, EVs emerge as crucial players in the brain and elucidating their physiological relevance opens up new perspectives in CNS research.

\section{ACKNOWLEDGMENTS}

The authors thank Jacqueline Trotter for support and discussion and Wiebke Möbius for providing the EM picture. Our work is supported by the Deutsche Forschungsgemeinschaft (KR 3668/1-1), and the Johannes Gutenberg University Mainz by the internal funding program and the Focus Program of Translational Neuroscience (FTN). 


\section{REFERENCES}

Al-Nedawi, K., Meehan, B., Micallef, J., Lhotak, V., May, L., Guha, A., et al. (2008). Intercellular transfer of the oncogenic receptor EGFRvIII by microvesicles derived from tumour cells. Nat. Cell Biol. 10, 619-624. doi: 10.1038/ncb1725

Antonucci, F., Turola, E., Riganti, L., Caleo, M., Gabrielli, M., Perrotta, C., et al. (2012). Microvesicles released from microglia stimulate synaptic activity via enhanced sphingolipid metabolism. EMBO J. 31, 1231-1240. doi: 10.1038/emboj. 2011.489

Baietti, M. F., Zhang, Z., Mortier, E., Melchior, A., Degeest, G., Geeraerts, A., et al. (2012). Syndecan-synteninALIX regulates the biogenesis of exosomes. Nat. Cell Biol. 14, 677-685. doi: 10.1038/ncb2502

Bakhti, M., Winter, C., and Simons, M. (2010). Inhibition of myelin membrane sheath formation by oligodendrocyte-derived exosomelike vesicles. J. Biol. Chem. 286, 787-796. doi: 10.1074/jbc.M110. 190009

Basso, M., Pozzi, S., Tortarolo, M., Fiordaliso, F., Bisighini, C., Pasetto, L., etal. (2013). Mutant copperzinc superoxide dismutase (SOD1) induces protein secretion pathway alterations and exosome release in astrocytes: implications for disease spreading and motor neuron pathology in amyotrophic lateral sclerosis. J. Biol. Chem. 288, 1569915711. doi: 10.1074/jbc.M112. 425066

Bellingham, S. A., Guo, B. B., Coleman, B. M., and Hill, A. F. (2012). Exosomes: vehicles for the transfer of toxic proteins associated with neurodegenerative diseases? Front. Physiol. 3:124. doi: 10.3389/fphys.2012.00124

Bianco, F., Perrotta, C., Novellino, L., Francolini, M., Riganti, L., Menna, E., et al. (2009). Acid sphingomyelinase activity triggers microparticle release from glial cells. $E M B O \mathrm{~J}$. 28, 1043-1054. doi: 10.1038/emboj. 2009.45

Bianco, F., Pravettoni, E., Colombo, A., Schenk, U., Moller, T., Matteoli, M., et al. (2005). Astrocyte-derived ATP induces vesicle shedding and IL-1 beta release from microglia. J. Immunol. 174, 7268-7277.

Bobrie, A., Colombo, M., Raposo, G., and Thery, C. (2011). Exosome secretion: molecular mechanisms and roles in immune responses. Traffic 12, 16591668. doi: 10.1111/j.1600-0854. 2011.01225.x
Buschow, S. I., Nolte-'t Hoen, E. N., van Niel, G., Pols, M. S., ten Broeke, T., Lauwen, M., et al. (2009). MHC II in dendritic cells is targeted to lysosomes or $\mathrm{T}$ cell-induced exosomes via distinct multivesicular body pathways. Traffic 10, 15281542. doi: 10.1111/j.1600-0854.2009. 00963.x

Ceruti, S., Colombo, L., Magni, G., Vigano, F., Boccazzi, M., Deli, M. A., et al. (2011). Oxygen-glucose deprivation increases the enzymatic activity and the microvesicle-mediated release of ectonucleotidases in the cells composing the blood-brain barrier. Neurochem. Int. 59, 259-271. doi: 10.1016/j.neuint.2011.05.013

Chivet, M., Hemming, F., PernetGallay, K., Fraboulet, S., and Sadoul, R. (2012). Emerging role of neuronal exosomes in the central nervous system. Front. Physiol. 3:145. doi: 10.3389/fphys.2012.00145

Chivet, M., Javalet, C., Hemming, F., Pernet-Gallay, K., Laulagnier, K., Fraboulet, S., et al. (2013). Exosomes as a novel way of interneuronal communication. Biochem. Soc. Trans. 41 241-244. doi: 10.1042/BST20120266 Cocucci, E., Racchetti, G., and Meldolesi, J. (2009). Shedding microvesicles: artefacts no more. Trends Cell Biol. 19, 43-51. doi: 10.1016/ j.tcb.2008.11.003

Colombo, E., Borgiani, B., Verderio, C., and Furlan, R. (2012). Microvesicles: novel biomarkers for neurological disorders. Front. Physiol. 3:63. doi: 10.3389/fphys.2012.00063

Falchi, A. M., Sogos, V., Saba, F., Piras, M., Congiu, T., and Piludu, M. (2013). Astrocytes shed large membrane vesicles that contain mitochondria, lipid droplets and ATP. Histochem. Cell Biol. 139, 221231. doi: 10.1007/s00418-0121045-x

Fitzner, D., Schnaars, M., Van Rossum, D., Krishnamoorthy, G., Dibaj, P., Bakhti, M., etal. (2011). Selective transfer of exosomes from oligodendrocytes to microglia by macropinocytosis. J. Cell Sci. 124, 447-458. doi: 10.1242/jcs.074088

Frühbeis, C., Fröhlich, D., and KrämerAlbers, E. M. (2012). Emerging roles of exosomes in neuron-glia communication. Front. Physiol. 3:119. doi: 10.3389/fphys.2012.00119

Frühbeis, C., Fröhlich, D., Kuo, W. P., Amphornrat, J., Thilemann, S., Saab, A. S., et al. (2013). Neurotransmitter-triggered transfer of exosomes mediates oligodendrocyte-neuron communication. PLoS Biol. 11:e1001604. doi: 10.1371/journal.pbio. 1001604
Fünfschilling, U., Supplie, L. M., Mahad, D., Boretius, S., Saab, A. S., Edgar, J., et al. (2012) Glycolytic oligodendrocytes maintain myelin and long-term axonal integrity. Nature 485, 517-521.

Gould, S. J., and Raposo, G. (2013). As we wait: coping with an imperfect nomenclature for extracellular vesicles. J. Extracell. Vesicles 2. doi: 10.342/jev.v2i0.20389

Gross, J. C., and Boutros, M. (2013). Secretion and extracellular space travel of Wnt proteins. Curr. Opin. Genet. Dev. 23, 385-390. doi: 10.1016/j.gde.2013.02.017

Gross, J. C., Chaudhary, V., Bartscherer, K., and Boutros, M. (2012). Active Wnt proteins are secreted on exosomes. Nat. Cell Biol. 14, 1036-1045. doi: $10.1038 /$ ncb2574

Hajrasouliha, A. R., Jiang, G., Lu, Q., Lu, H., Kaplan, H. J., Zhang, H. G., et al. (2013). Exosomes from retinal astrocytes contain anti-angiogenic components that inhibit laser-induced choroidal neovascularization. J. Biol. Chem. 288, 28058-28067. doi: 10.1074/jbc.M113.470765

Hanisch, U. K., and Kettenmann, H. (2007). Microglia: active sensor and versatile effector cells in the normal and pathologic brain. Nat. Neurosci. 10, 1387-1394. doi: 10.1038/ nn1997

Harding, C. V., Heuser, J. E., and Stahl, P. D. (2013). Exosomes: looking back three decades and into the future. J. Cell Biol. 200, 367-371. doi: $10.1083 /$ jcb. 201212113

Hooper, C., Sainz-Fuertes, R., Lynham, S., Hye, A., Killick, R., Warley, A., et al. (2012). Wnt3a induces exosome secretion from primary cultured rat microglia. BMC Neurosci. 13:144. doi: 10.1186/1471-220213-144

Hsu, C., Morohashi, Y., Yoshimura, S., Manrique-Hoyos, N., Jung, S., Lauterbach, M. A., et al. (2010). Regulation of exosome secretion by Rab35 and its GTPase-activating proteins TBC1D10A-C. J. Cell Biol. 189, 223-232. doi: $10.1083 / \mathrm{jcb}$. 200911018

Hu, G., Yao, H., Chaudhuri, A. D. Duan, M., Yelamanchili, S. V., Wen, H., et al. (2012). Exosome-mediated shuttling of microRNA-29 regulates HIV Tat and morphine-mediated neuronal dysfunction. Cell Death Dis. 3, e381. doi: 10.1038/cddis. 2012.114

Kalra, H., Simpson, R. J., Ji, H., Aikawa, E., Altevogt, P., Askenase, P., et al. (2012). Vesiclepedia: a compendium for extracellular vesicles with continuous community annotation. PLoS
Biol. 10:e1001450. doi: 10.1371/journal.pbio. 1001450

Koles, K., Nunnari, J., Korkut, C., Barria, R., Brewer, C., Li, Y., et al. (2012). Mechanism of evenness interrupted (Evi)-exosome release at synaptic boutons. J. Biol. Chem. 287, 16820-16834. doi: 10.1074/jbc.M112.342667

Korkut, C., Ataman, B., Ramachandran, P., Ashley, J., Barria, R., Gherbesi, N., et al. (2009). Trans-synaptic transmission of vesicular Wnt signals through Evi/Wntless. Cell 139, 393-404. doi: 10.1016/j.cell.2009. 07.051

Korkut, C., Li, Y., Koles, K., Brewer, C., Ashley, J., Yoshihara, M., et al. (2013). Regulation of postsynaptic retrograde signaling by presynaptic exosome release. Neuron 77, 10391046. doi: 10.1016/j.neuron.2013. 01.013

Krämer-Albers, E. M., Bretz, N., Tenzer, S., Winterstein, C., Möbius, W., Berger, H., etal. (2007). Oligodendrocytes secrete exosomes containing major myelin and stressprotective proteins: trophic support for axons? Proteomics Clin. Appl. 1, 1446-1461. doi: 10.1002/prca. 200700522

Lachenal, G., Pernet-Gallay, K., Chivet, M., Hemming, F. J., Belly, A., Bodon, G., et al. (2011). Release of exosomes from differentiated neurons and its regulation by synaptic glutamatergic activity. Mol. Cell. Neurosci. 46, 409-418. doi: 10.1016/j.mcn.2010. 11.004

Lee, Y., Morrison, B. M., Li, Y., Lengacher, S., Farah, M. H., Hoffman, P. N., et al. (2012). Oligodendroglia metabolically support axons and contribute to neurodegeneration. Nature 487, 443-448. doi: 10.1038 /nature 11314

Lewis, S. (2013). Glia: transporting cargo from A to B. Nat. Rev. Neurosci. 14, 589. doi: 10.1038/ nrn3568

Lopez-Verrilli, M. A., and Court, F. A. (2012). Transfer of vesicles from schwann cells to axons: a novel mechanism of communication in the peripheral nervous system. Front. Physiol. 3:205. doi: 10.3389/fphys.2012.00205

Luga, V., Zhang, L., Viloria-Petit, A. M., Ogunjimi, A. A., Inanlou, M. R., Chiu, E., et al. (2012). Exosomes mediate stromal mobilization of autocrine Wnt-PCP signaling in breast cancer cell migration. Cell 151, 1542-1556. doi: 10.1016/j.cell.2012.11.024

Nabhan, J. F., Hu, R., Oh, R S., Cohen, S. N., and Lu, Q. 
(2012). Formation and release of arrestin domain-containing protein 1-mediated microvesicles (ARMMs) at plasma membrane by recruitment of TSG101 protein. Proc. Natl. Acad. Sci. U.S.A. 109, 4146-4151.

Nave, K. A. (2010). Myelination and support of axonal integrity by glia. Nature 468, 244-252. doi: 10.1038/nature09614

Nave, K. A., and Trapp, B. D. (2008) Axon-glial signaling and the glial support of axon function. Annu. Rev. Neurosci. 31, 535-561. doi: 10.1146/ annurev.neuro.30.051606.094309

Ostrowski, M., Carmo, N. B., Krumeich, S., Fanget, I., Raposo, G., Savina, A., et al. (2010). Rab27a and Rab27b control different steps of the exosome secretion pathway. Nat. Cell Biol. 12, 19-30. doi: 10.1038/ ncb2000

Peinado, H., Aleckovic, M., Lavotshkin, S., Matei, I., Costa-Silva, B., MorenoBueno, G., et al. (2012). Melanoma exosomes educate bone marrow progenitor cells toward a prometastatic phenotype through MET. Nat. Med. 18, 883-891. doi: 10.1038/ nm. 2753

Potolicchio, I., Carven, G. J., Xu, X., Stipp, C., Riese, R. J., Stern, L. J., etal. (2005). Proteomic analysis of microglia-derived exosomes: metabolic role of the aminopeptidase CD13 in neuropeptide catabolism. J. Immunol. 175, 2237-2243.

Prada, I., Furlan, R., Matteoli, M., and Verderio, C. (2013). Classical and unconventional pathways of vesicular release in microglia. Glia 61, 1003-1017. doi: 10.1002/glia. 22497

Proia, P., Schiera, G., Mineo, M. Ingrassia, A. M., Santoro, G., Savettieri, G., et al. (2008). Astrocytes shed extracellular vesicles that contain fibroblast growth factor2 and vascular endothelial growth factor. Int. J. Mol. Med. 21, 63-67.
Raposo, G., and Stoorvogel, W (2013). Extracellular vesicles: exosomes, microvesicles, and friends. J. Cell Biol. 200, 373-383. doi: 10.1083/jcb.201211138

Saijo, K., and Glass, C. K. (2011). Microglial cell origin and phenotypes in health and disease. Nat. Rev. Immunol. 11, 775-787. doi: 10.1038/nri3086

Sbai, O., Ould-Yahoui, A., Ferhat, L., Gueye, Y., Bernard, A., Charrat, E. et al. (2010). Differential vesicular distribution and trafficking of MMP2, MMP-9, and their inhibitors in astrocytes. Glia 58, 344-366.

Schneider, A., and Simons, M. (2012). Exosomes: vesicular carriers for intercellular communication in neurodegenerative disorders. Cell Tissue Res. 352, 33-47 doi: 10.1007/s00441-012 $1428-2$

Sharma, P., Schiapparelli, L., and Cline, H. T. (2013). Exosomes function in cell-cell communication during brain circuit development. Curr. Opin. Neurobiol. doi: 10.1016/ j.conb.2013.08.005 [Epub ahead of print].

Simons, M., and Raposo, G. (2009). Exosomes - vesicular carriers for intercellular communication. Curr. Opin. Cell Biol. 21, 575-581. doi: 10.1016/j.ceb.2009.03.007

Skog, J., Wurdinger, T., Van Rijn, S., Meijer, D. H., Gainche, L. Sena-Esteves, M., etal. (2008). Glioblastoma microvesicles transport RNA and proteins that promote tumour growth and provide diagnostic biomarkers. Nat. Cell Biol. 10, 1470-1476. doi: 10.1038/ ncb 1800

Tamboli, I. Y., Barth, E., Christian, L., Siepmann, M., Kumar, S., Singh, S., et al. (2010). Statins promote the degradation of extracellular amyloid \{beta\}-peptide by microglia via stimulation of exosome-associated insulin-degrading enzyme (IDE) secretion. J. Biol. Chem. 285,
37405-37414. doi: $10.1074 / j b c$ M110.149468

Taylor, A. R., Robinson, M. B., Gifondorwa, D. J., Tytell, M., and Milligan, C. E. (2007). Regulation of heat shock protein 70 release in astrocytes: role of signaling kinases. Dev. Neurobiol. 67, 1815-1829. doi: 10.1002/dneu.20559

Thery, C. (2011). Exosomes: secreted vesicles and intercellular communications. F1000 Biol. Rep. 3, 15. doi 10.3410/B3-15

Thery, C., Ostrowski, M., and Segura, E. (2009). Membrane vesicles as conveyors of immune responses. Nat. Rev. Immunol. 9, 581-593. doi: $10.1038 /$ nri2567

Trajkovic, K., Hsu, C., Chiantia, S. Rajendran, L., Wenzel, D., Wieland, F., et al. (2008). Ceramide triggers budding of exosome vesicles into multivesicular endosomes. Science 319, 1244-1247. doi: 10.1126/science. 1153124

Turola, E., Furlan, R., Bianco, F., Matteoli, M., and Verderio, C. (2012). Microglial microvesicle secretion and intercellular signaling. Front. Physiol. 3:149. doi: 10.3389/fphys.2012 00149

Valadi, H., Ekstrom, K., Bossios, A., Sjostrand, M., Lee, J. J., and Lotvall, J. O. (2007). Exosome-mediated transfer of mRNAs and microRNAs is novel mechanism of genetic exchange between cells. Nat. Cell Biol. 9, 654 659. doi: $10.1038 /$ ncb1596

Verderio, C., Muzio, L., Turola, E. Bergami, A., Novellino, L., Ruffini, F., et al. (2012). Myeloid microvesicles are a marker and therapeutic target for neuroinflammation. Ann. Neurol. 72, 610-624. doi: 10.1002/ana.23627

Wang, G., Dinkins, M., He, Q., Zhu, G. Poirier, C., Campbell, A., et al. (2012). Astrocytes secrete exosomes enriched with proapoptotic ceramide and prostate apoptosis response 4 (PAR4): potential mechanism of apoptosis induction in Alzheimer disease (AD).
J. Biol. Chem. 287, 21384-21395. doi: 10.1074/jbc.M112.340513

Wang, S., Cesca, F., Loers, G., Schweizer, M., Buck, F., Benfenati, F., et al. (2011). Synapsin I is an oligomannose-carrying glycoprotein, acts as an oligomannosebinding lectin, and promotes neurite outgrowth and neuronal survival when released via glia-derived exosomes. J. Neurosci. 31, 7275-7290. doi: 10.1523/JNEUROSCI.6476-10. 2011

Zhang, Y., Liu, D., Chen, X., Li, J., Li, L., Bian, Z., et al. (2010). Secreted monocytic miR150 enhances targeted endothelial cell migration. Mol. Cell 39, 133144. doi: 10.1016/j.molcel.2010.06. 010

Conflict of Interest Statement: The authors declare that the research was conducted in the absence of any commercial or financial relationships that could be construed as a potential conflict of interest.

Received: 13 September 2013; accepted: 01 October 2013; published online: 30 October 2013.

Citation: Frühbeis C, Fröhlich D, Kuo WP and Krämer-Albers E-M (2013) Extracellular vesicles as mediators of neuron-glia communication. Front. Cell. Neurosci. 7:182. doi: 10.3389/fncel.2013.00182

This article was submitted to the journal Frontiers in Cellular Neuroscience.

Copyright (C) 2013 Frühbeis, Fröhlich, Kuo and Krämer-Albers. This is an openaccess article distributed under the terms of the Creative Commons Attribution License (CC BY). The use, distribution or reproduction in other forums is permitted, provided the original author(s) or licensor are credited and that the original publication in this journal is cited, in accordance with accepted academic practice. No use, distribution or reproduction is permitted which does not comply with these terms. 